\title{
A Comparison of Two Approaches to Three-Dimensional Imaging of Craniofacial Anomalies
}

\author{
Henry Rusinek, Nolan S. Karp, and Court B. Cutting
}

\begin{abstract}
Volume-based and surface-based algorithms for three-dimensional rendering of computed tomography (CT) scans of the human skull were compared in patients with craniofacial anomalies. Both methods were applied to a selected sample of 12 clinical CT studies. The number of sections ranged from 24 to 72 and the section thickness from 1.5 to $6.0 \mathrm{~mm}$. Volume renderings were more prone to interpolation artifacts but captured the anatomy in greater detail. The sites of closed cranial sutures, visualized using the volume technique, were not demonstrated using the specific surface rendering technique used in this study. In both techniques the areas of thin bone appeared as gaps.

(c) 1990 by W.B. Saunders Company.
\end{abstract}

KEY WORDS: computed tomography, image display, image processing, bones studies.

$\mathbf{T}$ ECHNIQUES for three-dimensional (3D) rendering of medical images can be categorized as surface-based or volume-based. In the surface approach the structure edges are extracted from the scan and mapped to some representation of the surface elements..$^{1-3}$ In the volume approach, the entire set of image data is projected along the parallel rays of view. ${ }^{4-7}$ The contribution of a voxel to the projected image depends on the user-defined opacity. The depth perception in both volume and surface techniques is enhanced by shading, usually derived from the angle between the spatial image gradient and the direction of light. ${ }^{8}$

Because the volume rendering method portrays the entire scan rather than a small subset corresponding to a fixed gray level, it is generally considered to yield a more detailed representation of the original data. ${ }^{9}$ However, the issue of superiority of the volume rendering is controversial and little is known about advantages and disadvantages of each technique in medical imaging. ${ }^{10}$

Previous clinical applications of volume rendering addressed the issues involving in imaging fractures. ${ }^{11-13}$ In this study we evaluated the ability to visualize craniofacial deformities on two commercial imaging systems, one based on the surface and the other the volume rendering method.

Three-dimensional rendering of complex, overlapping craniofacial structures can be valuable in planning craniofacial surgery. ${ }^{14-17}$ Potentially, a large number of axial computed tomography (CT) sections can be reduced to a few key views without loss of diagnostic information. 3D imaging is especially helpful in the preoperative assessment of patients with the following disorders: (1) craniosynostosis, both isolated and syndromal (Apert's, Crouzon's, and Pfeiffer's syndromes); (2) hypertelorism and orbital dystopia; (3) hemifacial microsomia; (4) bone tumors (most notably fibrous dysplasia) and soft tissue tumors with the associated effects on the craniofacial skeleton; and (5) displacements due to facial fractures.

The present study dealt with the application of 3D imaging to the first three types of anomalies. We evaluated artifacts associated with clinical images acquired with nonoverlapping sections of standard thickness. Acquiring a large number of 2- to 3-mm thin and overlapping CT sections may reduce the 3D display artifacts but it significantly increases the radiation dose. ${ }^{18}$ The increased radiation is of particular concern when dealing with young patients who, because of the smaller head size and of the potential need for multiple CT scans, will receive higher doses than the adults. ${ }^{19,20}$

We also evaluated the difficulty demonstrating the site of closed cranial sutures, loss of thin bone through partial volume averaging, step pattern artifacts associated with thick CT sections, ${ }^{21,22}$ and the accuracy of rendering existing gaps in bone as well as in artificially introduced edges.

From the Departments of Radiology and the Institute of Reconstructive Plastic Surgery, New York University Medical Center, New York, NY.

Address reprint requests to Henry Rusinek, PhD, Radiology Department, New York University Medical Center, 550 First Ave, New York, NY 10016.

- 1990 by W.B. Saunders Company.

0897-1889/90/0302-0003\$03.00/0 


\section{MATERIALS AND METHODS}

For the past 5 years we have used a surface-rendering system to plan craniofacial surgery and have now processed over $250 \mathrm{CT}$ examinations. The 3D images were generated on Cemax-1000 (software version 1.0; Cemax, Santa Clara, CA) and Cemax-5000 (software version 1.2) workstations in two steps. ${ }^{23}$ First, the contours of the bone were extracted from each plane based on a threshold value supplied by the user. At the same time, local vectors normal to the contours were computed. The threshold for bone was normally set to 175 Hounsfield units $(\mathrm{Hu})$ with lower limits used for young patients or those known to have thin bone. In the second step, using an arbitrary angle of view and light source, the images were rendered and shaded.

This volume-rendering system consisted of the Pixar image computer (Pixar, San Rafael, CA) and ChapVolumes (release 1.0), a package of volume-rendering subroutines. Except as noted previously, pixels in the range from 150 to 750 $\mathrm{Hu}$ were mapped onto the opacity range $0 \%$ to $100 \%$ using a linear transformation.

The CT scans were acquired on Philips 310 and TX60 scanners (Philips Medical Systems, Shelton, CT) and reconstructed into a $256 \times 256$ matrix. The scanners were set to 120 kilovolts (peak) and, depending on the need of the study, 220 to $440 \mathrm{~mA}$.

Twelve typical craniofacial studies (Table 1) previously imaged by the surface method were selected and processed using the volumetric technique. The number of sections varied from 24 to 72 . All CT sections were contiguous, with no overlapping or unsampled areas. Six CT scans were performed using a craniofacial protocol consisting of $1.5-\mathrm{mm}$ cuts through the orbits and $6.0-\mathrm{mm}$ cuts through the rest of the craniofacial skeleton; one scan was performed using uniform cut thickness of $4.0 \mathrm{~mm}$, two using $5.0 \mathrm{~mm}$, and three using uniform $6.0-\mathrm{mm}$ cuts through the entire craniofacial skeleton.

In two subjects, we made linear measurements of known thin $(<100 \mathrm{Hu})$ bone, which appeared as gaps. The maximum extent of the gaps in the anteroposterior direction was first marked with thin lines on computer monitor. The distance between the marks was measured in pixels on both the digital CT images and the 3D views obtained by the surface and the volume techniques. We also varied the

Table 1. Diagnoses of Patients in the Study

\begin{tabular}{rcl}
\hline Case No. & Age & \multicolumn{1}{c}{ Diagnoses } \\
\hline 1 & $13 \mathrm{yr}$ & Hemifacial microsomia \\
2 & $10 \mathrm{yr}$ & Crouzon's syndrome \\
3 & $11 \mathrm{yr}$ & Plagiocephaly \\
4 & $7 \mathrm{yr}$ & Hypertelorism \\
5 & $6 \mathrm{mo}$ & Pfeiffer's syndrome \\
6 & $16 \mathrm{mo}$ & Hemifacial microsomia, plagiocephaly \\
7 & $3.5 \mathrm{yr}$ & Trigonencephaly \\
8 & $5 \mathrm{mo}$ & Pfeiffer's syndrome \\
9 & $5 \mathrm{yr}$ & Plagiocephaly \\
10 & $22 \mathrm{yr}$ & Apert's syndrome \\
11 & $1 \mathrm{yr}$ & Median facial cleft \\
12 & $23 \mathrm{yr}$ & Apert's syndrome \\
\hline
\end{tabular}

threshold (surface method) and the range (volume method) of $\mathrm{Hu}$ to determine whether adjusting the settings has an effect on the size of the gap.

In an attempt to determine the ability of each technique to visualize small displacements, a 4-cm thick coronal section of the skull was shifted laterally 1 pixel, 2 pixels, and 4 pixels $(0.87,1.74$, and $3.48 \mathrm{~mm}$, respectively) in two scans. Each set of displaced images was rendered separately.

Images were shaded by a computer-generated light source placed to the right and in front of the subject. From each scan the right lateral, left lateral, frontal, superior, and inferior views were generated.

\section{RESULTS}

The volumetric renderings demonstrated the site of naturally closed cranial sutures in the nine patients old enough to have them. None of the sutures were demonstrated by the surfacerendering system used in this study. Three infants with open cranial sutures were accurately portrayed as open by both techniques.

Significant bone edge step pattern artifacts were present in all volume-rendered images with CT section thickness $5.0 \mathrm{~mm}$ or larger. These patterns were not discernible in surface-rendered images.

The detail in the presentation of the teeth was significantly improved in the volume-rendered images. Of the eight subjects 2 years of age or older, the volumetric method portrayed the number and location of the teeth in all patients. The representation of teeth by the surface-rendering technique had indistinct borders between teeth and unclear dental maxillary junctions.

In the two scans with the skull artificially displaced, the shift as small as 1 pixel was visible in both volume and surface images. The shift is shown as a pair of vertical lines in Figs $1 C$ and $D$.

Both techniques displayed 1- to 2-pixel thin bone of density less than $100 \mathrm{Hu}$ as gaps. The linear dimension of the gaps measured in the two-dimensional (2D) images (Fig 2A) agreed with the measurements from the $3 \mathrm{D}$ views to within 1 pixel $(0.87 \mathrm{~mm})$ in both volume and surface renderings. As illustrated in Fig 2B, extending the range of visible voxels to as low as $0 \mathrm{Hu}$ did not change the apparent size of the gap.

\section{REPRESENTATIVE CASES}

The following five cases illustrate the differences between the two techniques. The figures are presented in pairs: the surface-rendered im- 

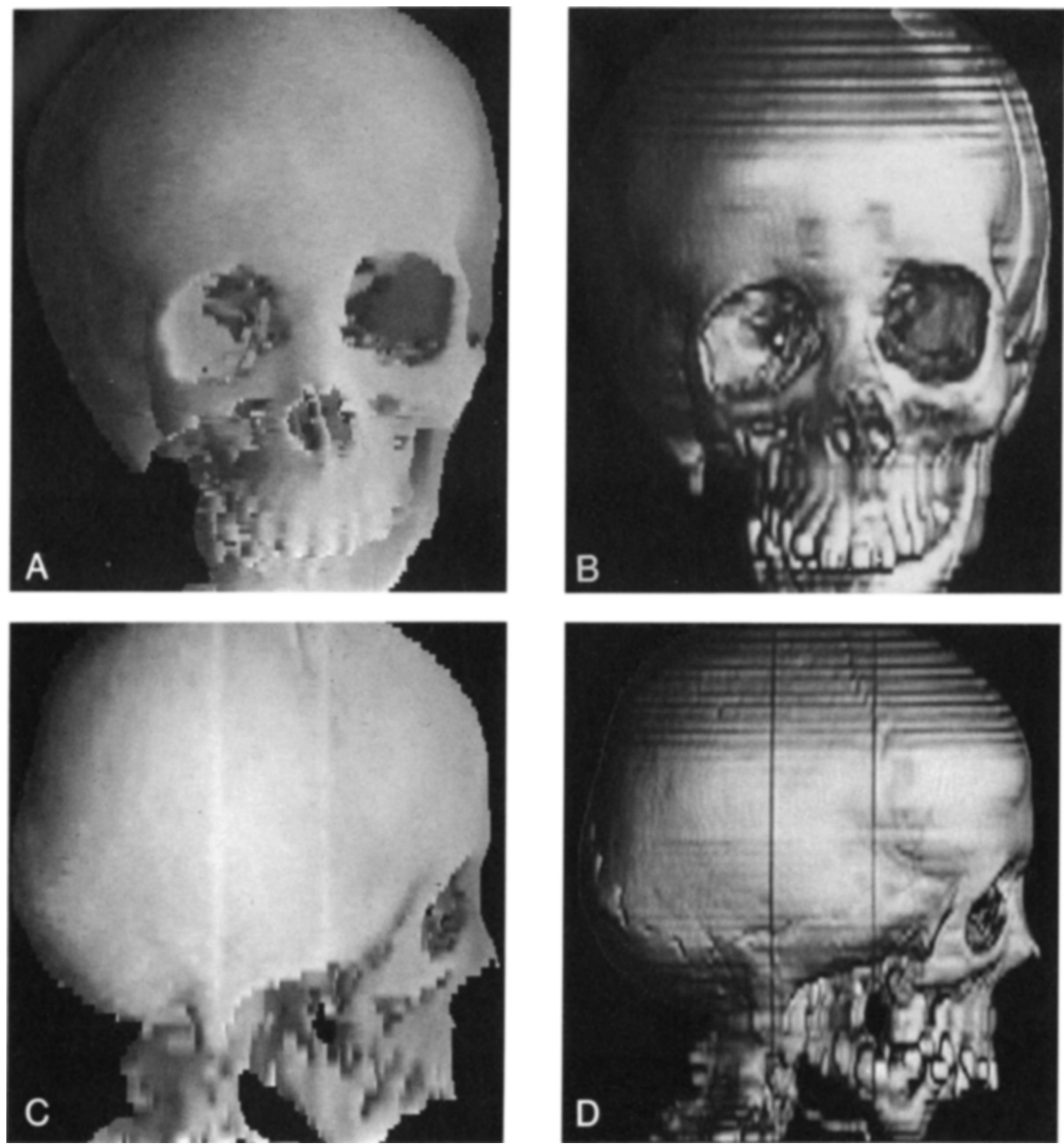

Fig 1. Frontal (A,B) and right lateral (C,D) views of a 13-year-old boy with hemifacial microsomia. Images were obtained using the (A,Cl surface-rendering method, and $(B, D)$ volume-rendering method. The coronal and lambdoid sutures are visible only on the volume-rendered image (D). The pair of vertical lines seen in images $C$ and $D$ represent an artificially introduced lateral shift of 1 pixel (approximately $1 \mathrm{~mm}$ ).

age on the left and the volume-rendered image on the right.

\section{Case I}

Case 1 involves a 13-year-old boy with hemifacial microsomia, hypoplasia of the mandible, and ipsilateral craniofacial skeleton. The preopera- tive scan consists of $56 \mathrm{CT}$ sections $1.5 \mathrm{~mm}$ thick in the area of the orbits and $6.0 \mathrm{~mm}$ for the rest of the craniofacial skeleton. Images were acquired on Philips 60TX scanner set at $120 \mathrm{kVp}$ and 440 $\mathrm{mA}$. In the frontal view (Figs $1 \mathrm{~A}$ and $\mathrm{B}$ ) the underdevelopment of the right mandible is clearly visible in both images. The volume-rendered 

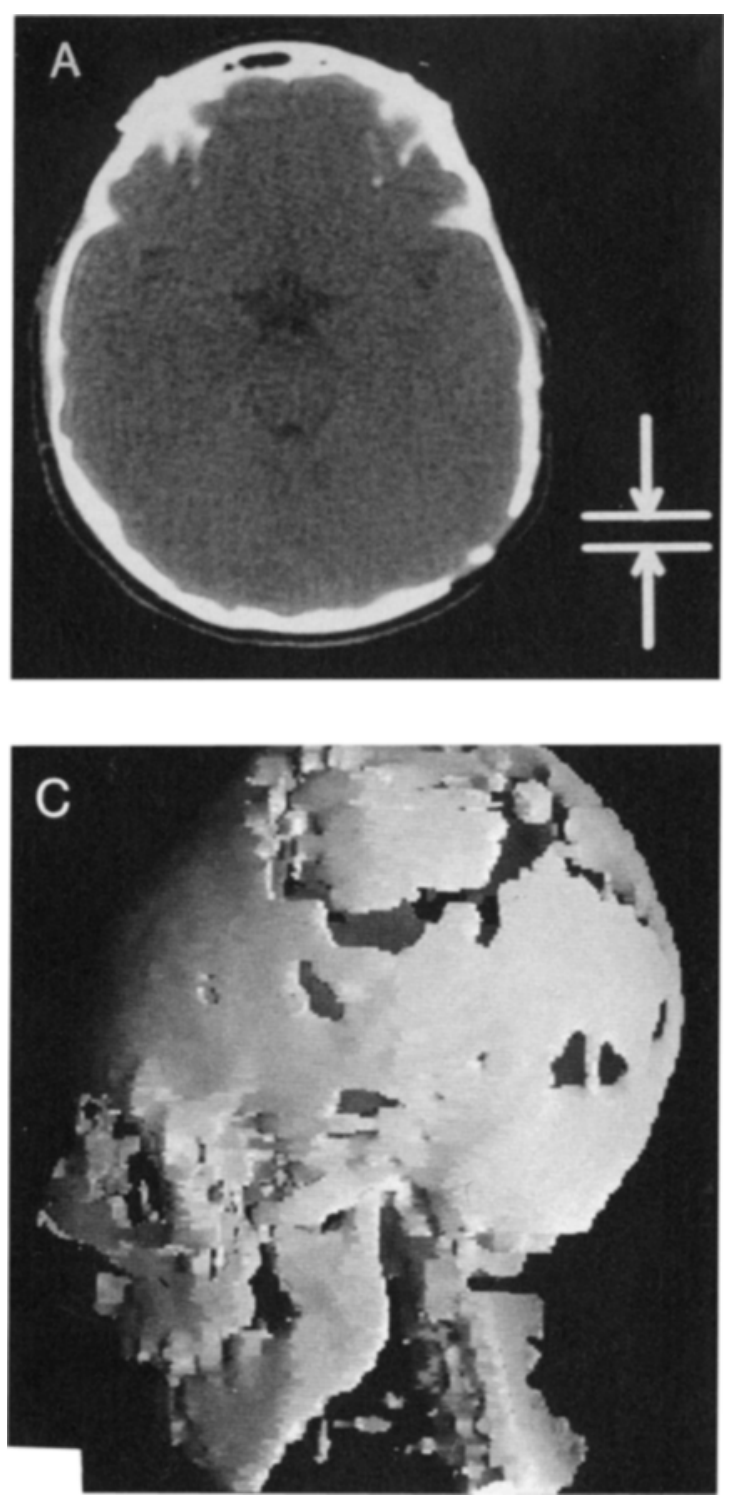

image (Fig 1B) shows increased overall detail. In the right lateral view (Figs $1 C$ and D) the closed coronal and lambdoid sutures are visible only on the volume rendering.

\section{Case 2}

Case 2 (Fig 2) involves a 10-year-old girl with Crouzon's syndrome, craniofacial dystosis, who had frontal bone advancement and cranial vault remodeling as an infant. The CT scan was performed in preparation for a second operation. The scan consisted of 38 6-mm thick sections acquired on Philips 310 scanner using $120 \mathrm{kVp}$ and $350 \mathrm{~mA}$ technique. This case was chosen to compare imaging of thin bone. The presence of

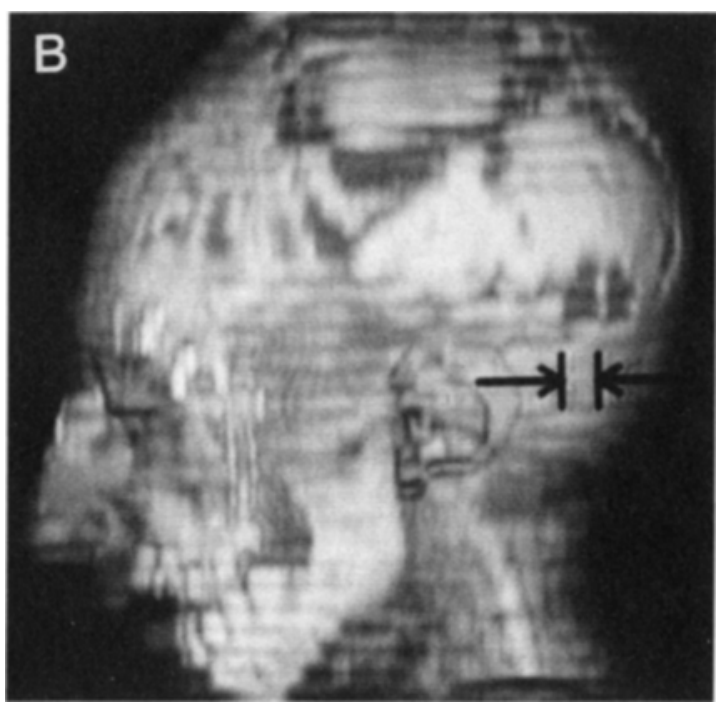

Fig 2. (A) A gap consisting of thin ( $<100 \mathrm{Hu}$ ) bone measured in a CT section from a 10-year-old girl with Crouzon's syndrome. (B) The corresponding measurement in a volume rendering using an extended gray density range of $\mathrm{O}$ to $750 \mathrm{Hu}$. (C) Surface rendering.

bone in this subject has been established by clinical examination as well as by an analysis of the pixel density in the original CT scan. Both volume- and surface-rendered images in Figs 2B and $C$ show large areas of missing bone.

\section{Case 3}

Case 3 involves an 11-year-old girl with unilateral coronal synostosis (plagiocephaly) who at 6 months of age underwent right frontal bone advancement via an intracranial approach. The CT scan consists of 62 sections $1.5 \mathrm{~mm}$ thick in the area of the orbits and $6.0 \mathrm{~mm}$ for the rest of the craniofacial skeleton. Images were acquired on Philips 60TX scanner set at $120 \mathrm{kVp}$ and 440 
mA. On both images (Fig 3) the deformity of the right frontal bone and the postsurgical changes are readily apparent. The volumetric rendering (Fig 3B) appears more detailed, with individual teeth and postsurgical wires resolved better than on the surface-rendered image. However, in the volumetric image the top of the skull forms step edges.

\section{Case 4}

Case 4 involves a 7 -year-old boy with orbital hypertelorism. The CT study consists of 32 $5.0-\mathrm{mm}$ thick sections acquired on Philips 310 scanner using $120 \mathrm{kVp}$ and $400 \mathrm{~mA}$ technique. The frontal view (Fig 4) demonstrates the increased distance between the orbits. In both images the right orbit appears unnaturally bright. The volume rendering shows the relationship of the teeth to the facial bones with greater detail.

\section{Case 5}

Case 5 involves a 6-month-old infant with Pfeiffer's syndrome and multiple suture synostoses. The CT scan (Philips $310 \mathrm{CT}, 120 \mathrm{kVp}$, $220 \mathrm{~mA}$ ), performed prior to cranial vault remodeling and advancement of the frontal bone, con-

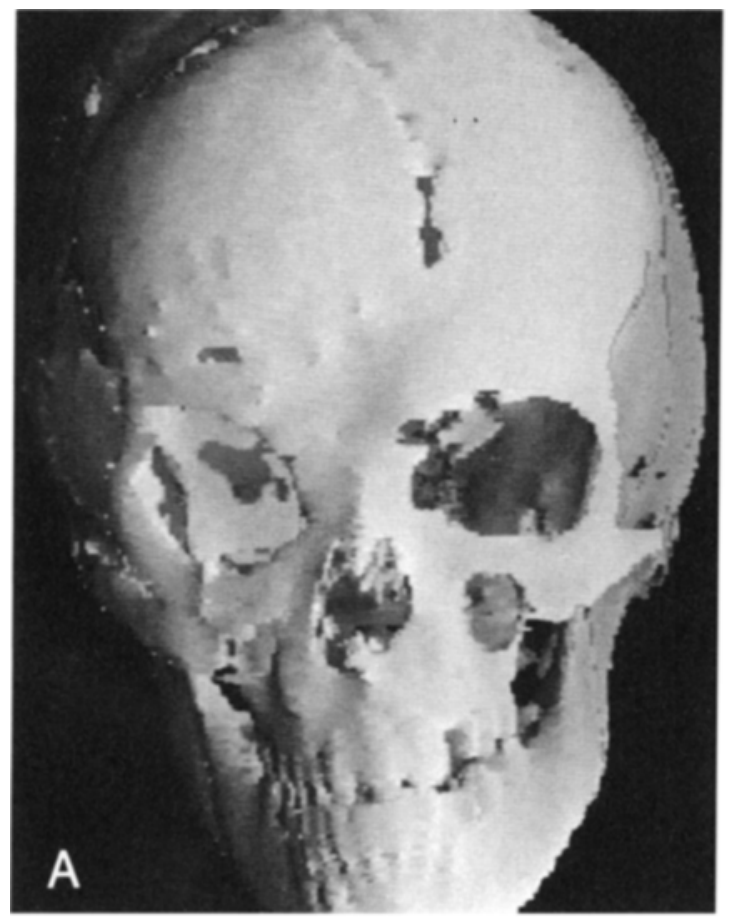

sists of 25 sections $5.0 \mathrm{~mm}$ thick. In the 3D views (Fig 5) the sutures appear open and bulging. The superior portion of the skull is discontinuous. Even though both images suffer from artifacts, the surface-rendered image maintains some detail whereas the volumetric image is more difficult to interpret.

\section{DISCUSSION}

The volumetric method showed the anatomy in greater detail in scans with $1.5-\mathrm{mm}$ to $4.0-\mathrm{mm}$ thin CT sections. We have not observed unnatural effects due to translucent bone. Although the surface extraction method used in this study could capture a regular ( $<1-\mathrm{mm}$ deep) indentation in the skull (Figs $1 C$ and D), irregular features such as postsurgical wires, cranial sutures, and teeth were seen less clearly or, in the case of closed cranial sutures, not visualized at all. Because more accurate and faster surface extraction algorithms have recently been developed, ${ }^{24,25}$ we emphasize that our observations are applicable only to the two commercial imaging systems used in this study.

The volumetric technique was found to be more sensitive to step pattern artifacts associated

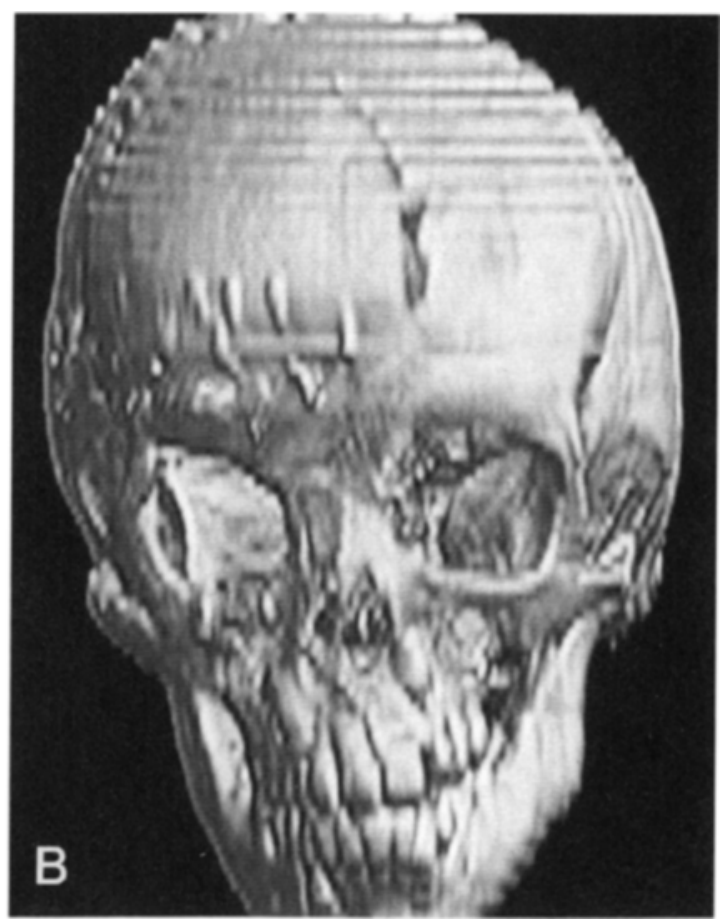

Fig 3. Frontal view of a 11-year-oid girl after a right frontal bone advancement: (A) surface-rendered image, (B) volume-rendered image. Note better resolution of teeth and postsurgical wires on the volume-rendered image. 

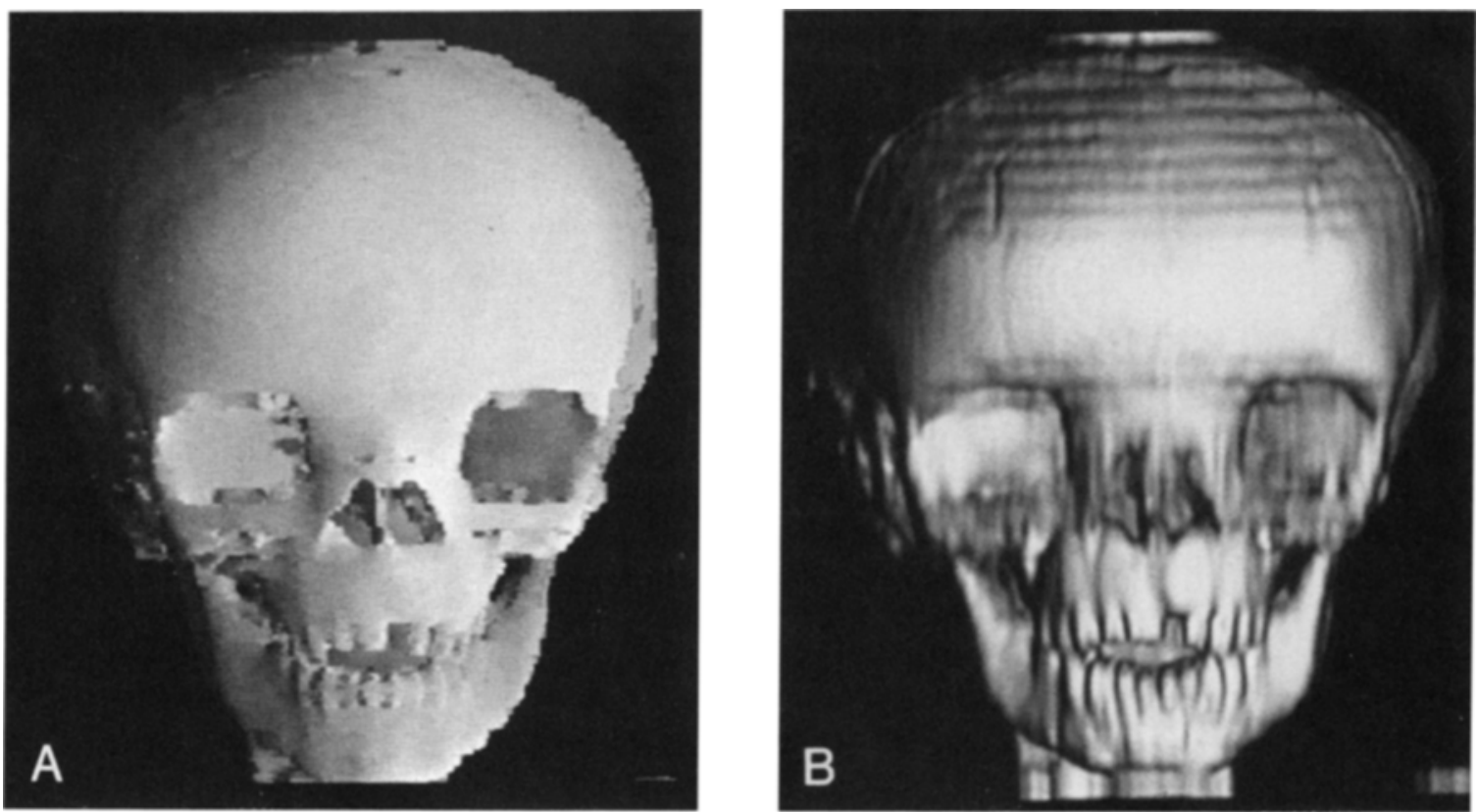

Fig 4. A 7-year-old boy with orbital hypertelorism. Both (A) surface rendering and (B) volume rendering demonstrate the increased distance between the orbits. The volume rendering shows the relationship of the teeth to the facial bones with greater detail. Note the brighter appearance of the right orbit. caused by unnatural light shading used by both rendering techniques.

with 5- to 6-mm thick CT sections and most pronounced in areas of large curvature, such as the top of the calvarium. This artifact is caused by the linear interpolation step used in this volume-rendering system. Figure 6 demonstrates reduced density of bone obtained by the straightforward linear interpolation and indicates the need and importance of improved algorithms for intensity interpolation.

The thin bone artifact depicted in Fig 2 could not be eliminated by decreasing the lower limit of the opacity range from the normally used value of $150 \mathrm{Hu}$. Bone appears discontinuous in the volume-rendered images because the pixel-thin layer of slightly increased density is not sufficient to make a visible contribution.

For simplicity of implementation, none of the methods generate shadows. For example, the right orbit in Fig 4 is unnaturally bright. Shadows can be correctly rendered by using an appropriate form of the ray tracing algorithm. Although it increases visual realism, ray tracing is very costly in computer time. ${ }^{26}$

The two algorithms differ in computer time and memory requirements. During the surface extraction a $10-$ to 20 -Mbyte volume of data is typically reduced by a factor of 10 . This process takes approximately 15 minutes on the Cemax5000 workstation. On the other hand, the image rendering step is rapid. Neither of the two steps require large memory. Interaction with data, eg, editing away objects obstructing the region of interest or changing the threshold density for the bone, may require extraction of totally new edges in surface-based algorithm, whereas it imposes no such time penalty in volume rendering.

Clearly, the number of computations needed to rotate, project, and shade is greater for the entire volume of the scan than for the extracted surface. In addition, because the volumetric method preserves all data, larger main memory is required. With current software the Pixar computer requires 20 minutes to generate 64 views from a scan made of 45 sections resolved into $256 \times 256$ pixels. Generating a single view takes 2 minutes because of the fixed overhead of data transfer and interpolation.

An important visual clue is conveyed by dynamic projections, or rapid display of a series of renderings, each from a slightly different viewpoint. For example, by rotating an object, points near the center of rotation appear to move slower than points away from the center, providing an added depth cue. We have restricted the present 

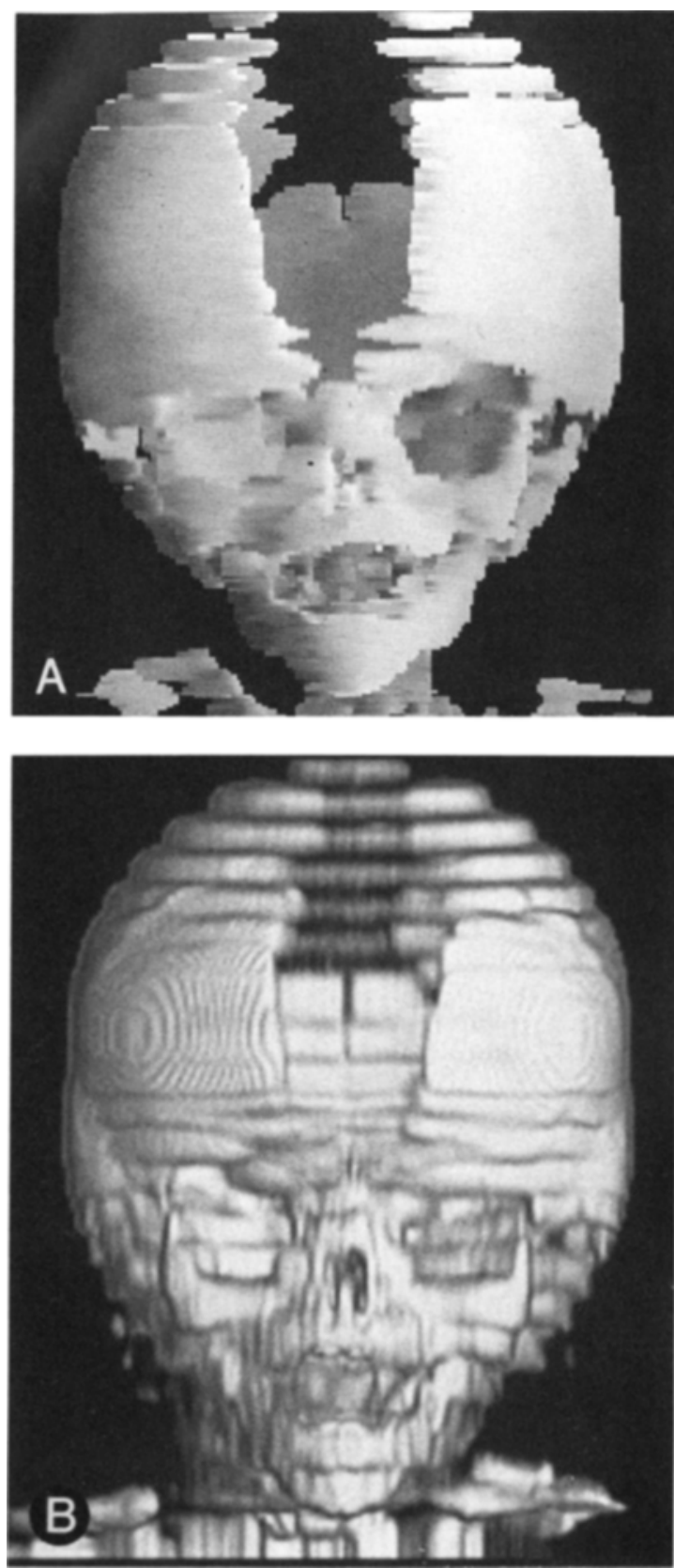

Fig 6. Images from a 6-month-old infant with Pfeiffer's syndrome: (A) surface, and (B) volume rendering. The CT scan consisted of 25 sections $5.0 \mathrm{~mm}$ thick. Both images are hampered by step pattern artifacts.

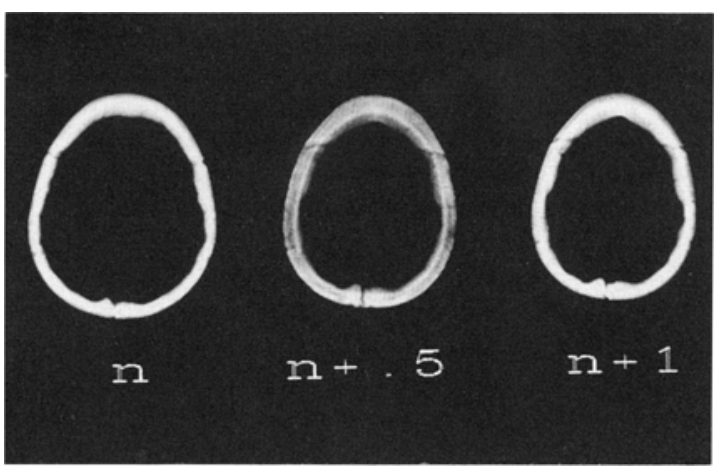

Fig 6. The artifacts in volume-rendering technique are caused by the interpolation step. In the middle image, obtained by interpolating from levels $n$ (left) and $n+1$ (right) to the level $n+0.6$, the bone exhibits decreased gray level and will appear dimmer.

study to static, or "hard copy" images. Of the two systems used in this study, only the volumerendering system is equipped with the video hardware for rapid dynamic display. In our experience, the dynamic projections are helpful in understanding the overall structure but do not help to resolve the detail. In addition, static images are necessary to take measurements and are easily portable for use in the operating room.

In the future we see well-defined, complementary roles for surface and volume imaging. Surface extraction is the first step in establishing a geometric hierarchy (ie, points, faces, ridges) within the data. Surface recognition will remain essential in applications involving fabrication of prostheses, feature identification, and measurement of anatomic landmarks. The potential of volume rendering lies in the ease in handling multiple tissues simultaneously, the ability to visualize objects in the presence of nonuniform signal, and in applications requiring gray scale analysis.

\section{ACKNOWLEDGMENT}

We thank Martha Helmers and Anthony Jalandoni for their assistance in preparing the illustrations.

\section{REFERENCES}

1. Herman GT, Liu HK: Display of three-dimensional information in computed tomography. $\mathrm{J}$ Comput Assist Tomogr 1:155-160, 1977

2. Ganapathy S, Dennehy TD: A new general triangulation method for planar contours. Comput Graphics 16:69-75, 1982
3. Cook LT, Dwyer SJ, Batnitsky S, et al: A threedimensional display system for diagnostic imaging. IEEE Comput Graphics Applic 3:13-19, 1983

4. Frieder G, Gordon D, Reynolds RA: Back-to-front display of voxel-based objects. IEEE Comput Graphics Applic 5:52-60, 1985 
5. Talton DA, Goldwasser SM, Reynolds RA, et al: Volume rendering algorithm for the presentation of $3 \mathrm{D}$ medical data. Proc Nat Comput Graphics Assoc 3:119-128, 1987

6. Drebin RA, Carpenter L, Hanrahan P: Volume rendering. Comput Graphics 22:65-74, 1988

7. Levoy M: Display of surfaces from volume data. IEEE Comput Graphics Applic 8:29-37, 1988

8. Hoehne KH, Bernstein R: Shading 3D images from CT using gray-level gradients. IEEE Trans Med Imaging MI-5: 45-47, 1986

9. Frenkel KA: Volume rendering. Commun Assoc Comput Machinery 32:426-435, 1989

10. Udupa JK, Herman GT: Volume rendering versus surface rendering. Commun Assoc Comput Machinery 32: 1364-1366, 1989

11. Fishman EK, Drebin RA, Magid D, et al: Volumetric rendering techniques: Applications for three-dimensional imaging of the hip. Radiology 163:737-738, 1987

12. Scott WW Jr, Fishman EK, Magid D: Acetabular fractures: Optimal imaging. Radiology 165:537-539, 1987

13. Drebin RA, Magid D, Robertson DD, et al: Fidelity of three-dimensional CT imaging for detecting fracture gaps. J Comput Assist Tomogr 13:487-489, 1989

14. Vannier MW, Marsh JL, Warren JO: Three-dimensional computer graphics for craniofacial surgical planning and evaluation. Comput Graphics 17:263-273, 1983

15. Hemmy DC, David DJ, Herman GT: Three-dimensional reconstruction of craniofacial deformity using computer tomography. Neurosurgery 13:534-541, 1983

16. Cutting C, Bookstein FL, Grayson B, et al: Threedimensional computer-assisted design of craniofacial surgical procedures: Optimization and interaction with cephalometric and CT-based models. Plast Reconstr Surg 77:877-885, 1986
17. Vannier MW, Hildebolt CF, Marsh JL, et al: Craniosynostosis: Diagnostic value of three-dimensional CT reconstruction. Radiology 173:669-673, 1989

18. McCrohan JL, Patterson JF, Gagne RM, et al: Average radiation doses in a standard head examination for 250 CT systems. Radiology 163:263-268, 1987

19. Fearon T, Vucich J: Pediatric patient exposures from CT examinations: GE CT/T 9800 scanner. AJR 144:805809,1985

20. Lassen MN: Dedicated CT technique for scanning neonates. Radiology 161:363-366, 1986

21. Vannier W, Marsh JL, Knapp RH, et al: Recent advances in 3-D CT scan reconstruction for clinical applications, in Lemke HU, Rhodes ML, Jaffee CC, et al (eds): Computer Assisted Radiology. New York, NY, SpringerVerlag, 1987, pp 657-661

22. Hemmy DC, Tessier PL: CT of dry skulls with craniofacial deformities: Accuracy of three-dimensional reconstruction. Radiology 157:113-116, 1985

23. Fellingham LL, Vogel $\mathbf{J H}$, Lau $\mathbf{C}$, et al: Interactive graphics and 3-D modelling for surgical planning and prosthesis and implant design. Proc Natl Comput Graphics Assoc 3:132-142, 1986

24. Lorensen WE, Cline HE: Marching cubes: A high resolution 3D surface construction algorithm. Comput Graphics 6:163-169, 1987

25. Chuang KS, Udupa JK, Raya SP: High-quality rendering of discrete three-dimensional surfaces. Tech Rep MIPG 130. Philadelphia, PA, Medical Image Processing Group, Department of Radiology, University of Pennsylvania, 1988

26. Kay T, Kajiya J: Ray tracing complex scenes. Comput Graphics 20:169-278, 1986 